\title{
Beam emittance control by changing injection painting area in a pulse-to-pulse mode in the 3-GeV rapid cycling synchrotron of Japan Proton Accelerator Research Complex
}

\author{
P. K. Saha, ${ }^{*}$ H. Harada, N. Hayashi, K. Horino, H. Hotchi, M. Kinsho, T. Takayanagi, N. Tani, T. Togashi, \\ T. Ueno, and Y. Yamazaki \\ Japan Atomic Energy Agency (JAEA), Tokai Naka, Ibaraki 319-1195, Japan \\ Y. Irie $^{\dagger}$ \\ High Energy Accelerator Research Organization (KEK), Tsukuba, Ibaraki 305-0801, Japan
}

(Received 12 August 2013; published 9 December 2013)

\begin{abstract}
The 3-GeV rapid cycling synchrotron (RCS) of Japan Proton Accelerator Research Complex (J-PARC) simultaneously delivers high intensity beam to the Material and Life Science Experimental Facility (MLF) as well as to the main ring (MR) at a repetition rate of $25 \mathrm{~Hz}$. The RCS is designed for a beam power of $1 \mathrm{MW}$. RCS has to meet not only the need of power upgrade but also the specific requirement of each downstream facility. One of the issues, especially for high intensity operation, is to maintain two different transverse sizes of the extracted beam for MLF and MR; namely, a wider beam for MLF in order to reduce damage on the neutron production target but reversely a narrower one for the MR in order to ensure a permissible beam loss in the beam transport line of $3-\mathrm{GeV}$ to MR and also in the MR. We proposed pulseto-pulse direct control of the transverse painting area during the RCS beam injection process in order to get an extracted beam profile as desired. In addition to two existing dc septum magnets used for fixing injected beam trajectory for MLF beam, two additional dipoles named pulse steering magnets are designed for that purpose in order to control injected beam trajectory for a smaller painting area for the MR. The magnets are already installed in the injection beam transport line and successfully commissioned well in advance before they will be put in normal operation in 2014 for the $400 \mathrm{MeV}$ injected beam energy upgraded from that of the present $181 \mathrm{MeV}$. Their parameters are found to be consistent to those expected in the corresponding numerical simulations. A trial one cycle user operation run for a painting area of $100 \pi \mathrm{mm}$ mrad for the MR switching from the MLF painting area of $150 \pi \mathrm{mm}$ mrad has also been successfully carried out. The extracted beam profile for the MR is measured to be sufficiently narrower as compared to that for the MLF, consistent with numerical simulation successfully demonstrating validity of the present principle.
\end{abstract}

DOI: 10.1103/PhysRevSTAB.16.120102

PACS numbers: 29.20.dk, 29.27.Ac

\section{INTRODUCTION}

The 3-GeV rapid cycling synchrotron (RCS) of Japan Proton Accelerator Research Complex (J-PARC) is designed for a high power proton beam source of $1 \mathrm{MW}$ $\left(8.33 \times 10^{13}\right.$ protons per pulse $)$ for the neutron and muon production targets in the Material and Life Science Experimental Facility (MLF) as well as an injector for the $50-\mathrm{GeV}$ main ring synchrotron (MR) [1]. Figure 1 shows a schematic view of the RCS, which is a threefold symmetric lattice having a circumference of $348.333 \mathrm{~m}$.

\footnotetext{
*Corresponding author.

saha.pranab@j-parc.jp

${ }^{\dagger}$ Present address: Institute of High Energy Physics (IHEP), Beijing, China.

Published by the American Physical Society under the terms of the Creative Commons Attribution 3.0 License. Further distribution of this work must maintain attribution to the author(s) and the published article's title, journal citation, and DOI.
}

Each superperiod consists of two 3-DOFO (defocusing and focusing periodic cell structure) arc modules with missing bends and 3-DOFO insertions. The maximum dispersion at the arc (missing bends) is about $6 \mathrm{~m}$, while the insertions are dispersion free. The injection and transverse collimation systems are located in the first insertion. The injected beam energy is $181 \mathrm{MeV}$ at present but it will be upgraded to the designed $400 \mathrm{MeV}$ in 2013. The extracted beam energy is $3 \mathrm{GeV}$ and is simultaneously delivered to the MLF and MR at a repetition rate of $25 \mathrm{~Hz}$. A pattern dipole magnet named pulse bending (PB) magnet located downstream of the RCS extraction beam transport (BT) line is used to switch the beam destination to the MR according to the operation strategy. The beam power of the RCS for the user operation has already exceeded $300 \mathrm{~kW}$ and feasibility of further increase, exceeding $500 \mathrm{~kW}$ operation, has also been demonstrated in a recent beam study $[2,3]$.

The designed goal of the RCS is not only to achieve a beam power of $1 \mathrm{MW}$ but also to ensure specific 


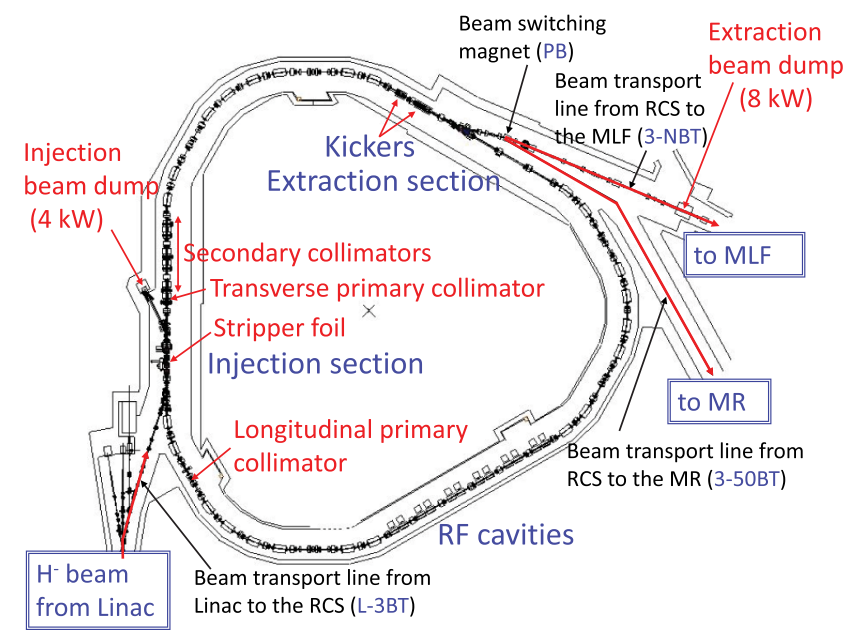

FIG. 1. Schematic view of the $3-\mathrm{GeV}$ RCS of J-PARC. Extracted beam is delivered to both the MLF and MR with time sharing at a repetition rate of $25 \mathrm{~Hz}$. A pattern dipole magnet named pulse bending magnet $(\mathrm{PB})$ located downstream of the RCS extraction line acts as a switching magnet for changing beam destination MLF to MR according to the operation strategy.

requirements of each downstream facility. One such issue is to maintain two different transverse sizes of the extracted beam for MLF and MR in simultaneous operation; namely, a sufficiently wider beam for MLF in order to reduce damage on the neutron production target but reversely a narrower one for the MR in order to ensure a permissible beam loss in the beam transport line of $3-\mathrm{GeV}$ RCS to the 50-GeV MR (3-50BT) as well as in the MR. RCS utilizes multiturn $\mathrm{H}^{-}$charge-exchange injection technique during beam accumulation of $0.5 \mathrm{~ms}$ and in order to mitigate space charge effect at the lower energy, both transverse and longitudinal injection painting have been successfully utilized [4-6]. In the present study, we proposed pulse-to-pulse direct control of the transverse painting area during the injection process in order to ensure a desired transverse beam profile or in other words, to ensure a desired transverse emittance at the extraction. For example, we considered a designed maximum feasible painting area of $216 \pi \mathrm{mm}$ mrad for the MLF but a relatively smaller painting area of $144 \pi \mathrm{mmmrad}$ for the MR.

Figure 2 shows an expected transverse rms emittance damping and dependence of the rms emittance at the end of acceleration on the initial injection painting area of 216,150 , and $100 \pi \mathrm{mm}$ mrads depicted by blue, black, and red lines, respectively. The painting emittance values are $99.7 \%$ emittance values for all cases. The simulation was done for the present injected beam energy of $181 \mathrm{MeV}$ and for a beam power of $350 \mathrm{~kW}$ at the extraction energy of $3 \mathrm{GeV}$, where a full 3D space charge effect was taken into account. It can be easily seen that a smaller initial painting area guarantees a comparatively

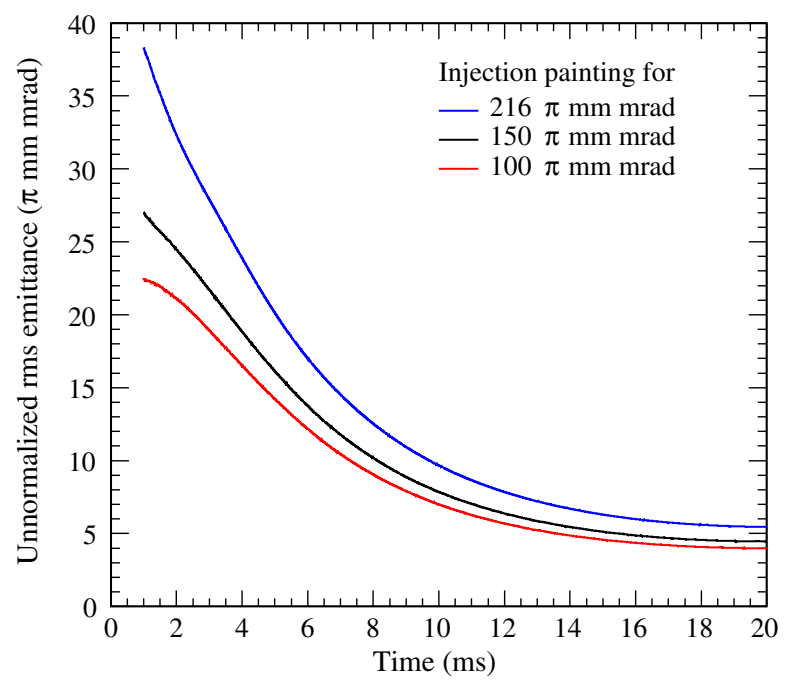

FIG. 2. Damping of the transverse rms horizontal emittance and dependence of the rms emittance at the top energy on the initial injection painting area as estimated in the numerical simulations. A smaller initial painting area gives a comparatively smaller emittance of the extracted beam.

smaller emittance of the extracted beam. One can expect nearly $25 \%$ reduction of an rms emittance for an injection painting area of $100 \pi \mathrm{mm}$ mrad as compared to that of $216 \pi \mathrm{mm}$ mrad.

However, in order to switch the painting area MLF to MR in a pulse-to-pulse mode, one accordingly has to changed the injected beam trajectory. As a result, two horizontal pulse dipole magnets are additionally proposed to install with existing two dc septum magnets (ISEP1-2). They are named pulse steering (PSTR) magnets and have already been installed in the linac to the $3-\mathrm{GeV}$ RCS beam transport (L-3BT) line in the 2012 summer shutdown period. Figure 3[7] shows a layout of the RCS injection area, where location of the newly installed PSTR magnets is shown by two blue arrows. A further schematic and expanded view of the injection area together with injected beam trajectory and the circulating orbit bump offset for injection painting is shown in Fig. 4. The PSTR1 is located at the upstream ISEP1, while PSTR2 is located between ISEP1 and ISEP2. The incoming $\mathrm{H}^{-}$beam from the linac is stripped to $\mathrm{H}^{+}$by the first stripper foil named first foil placed in the middle of four chicane bump magnets (SB1-4). The location of the first foil is also often mentioned as the injection point. The injected beam trajectory or more specifically the centroid of the injected beam at the injection point in the horizontal direction for MLF painting is determined by two septa (ISEP1-2), where PSTR1-2 are additionally used for MR painting in order to change only the angle of the injection beam to a smaller value by keeping its position unchanged at the injection point. A smaller injection angle with no change in position makes a smaller painting area for the MR. The detail is given in the next section. 


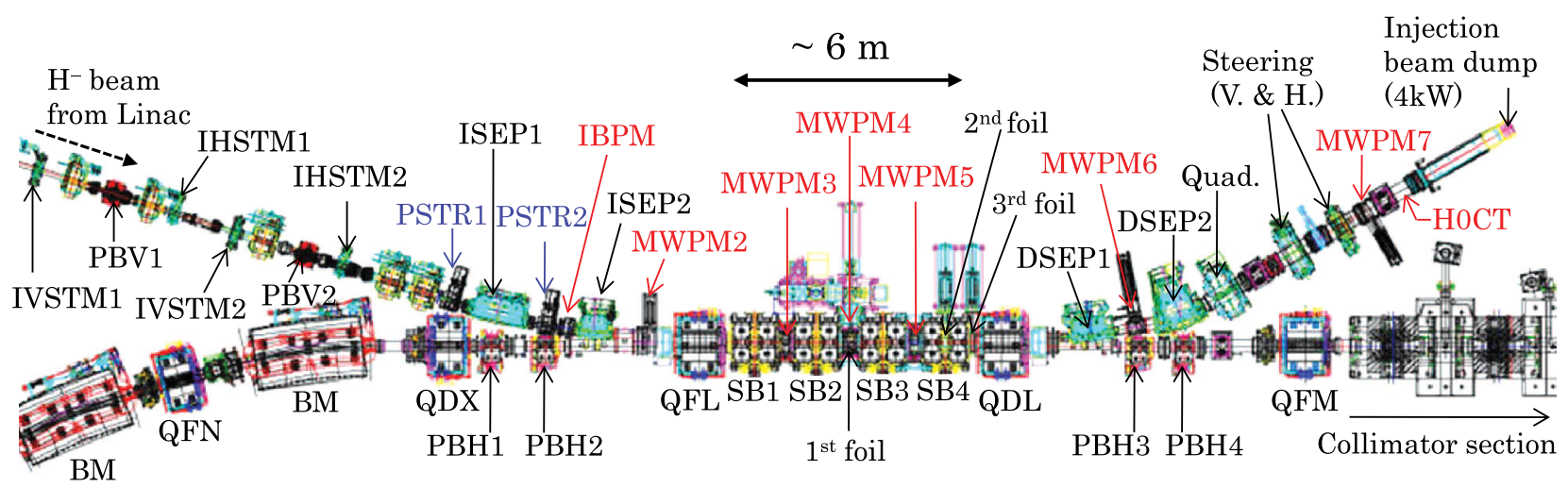

FIG. 3. Layout of RCS injection area. In order to switch transverse painting area MLF to MR in the horizontal direction, two PSTR magnets are recently installed in the L-3BT line as shown by blue arrows. The phase space coordinates of the injected beam at the first stripper foil in controlled pulse to pulse for that purpose. Four chicane bump magnets are named SB1-4, while four horizontal and two vertical painting bump magnets are named PBH1-4 and PBV1-2, respectively. The PBV1-2 are located in the L-3BT line and thus painting area in the vertical direction is also changed in a pulse to pulse. More than $99.6 \%$ of incoming $\mathrm{H}^{-}$beam from the linac is stripped to $\mathrm{H}^{+}$by the first foil so as to inject into the ring [7].

\section{PRINCIPLES AND DESIGN SPECIFICATIONS OF THE PSTR MAGNETS}

In order to mitigate the space charge effect at the lower energy, a uniform beam distribution is obtained by performing both transverse and longitudinal injection painting in the RCS. The importance and technique of the injection painting, as well as application to the real machine for reducing significant beam losses and achieving a high intensity beam has already been extensively presented in our earlier paper [6].

Figure 5 shows a schematic view of the transverse injection painting process performed in the RCS. The upper figure is for painting in the horizontal plane, while the lower one is that for the vertical direction. In the horizontal direction, the position and angular displacement

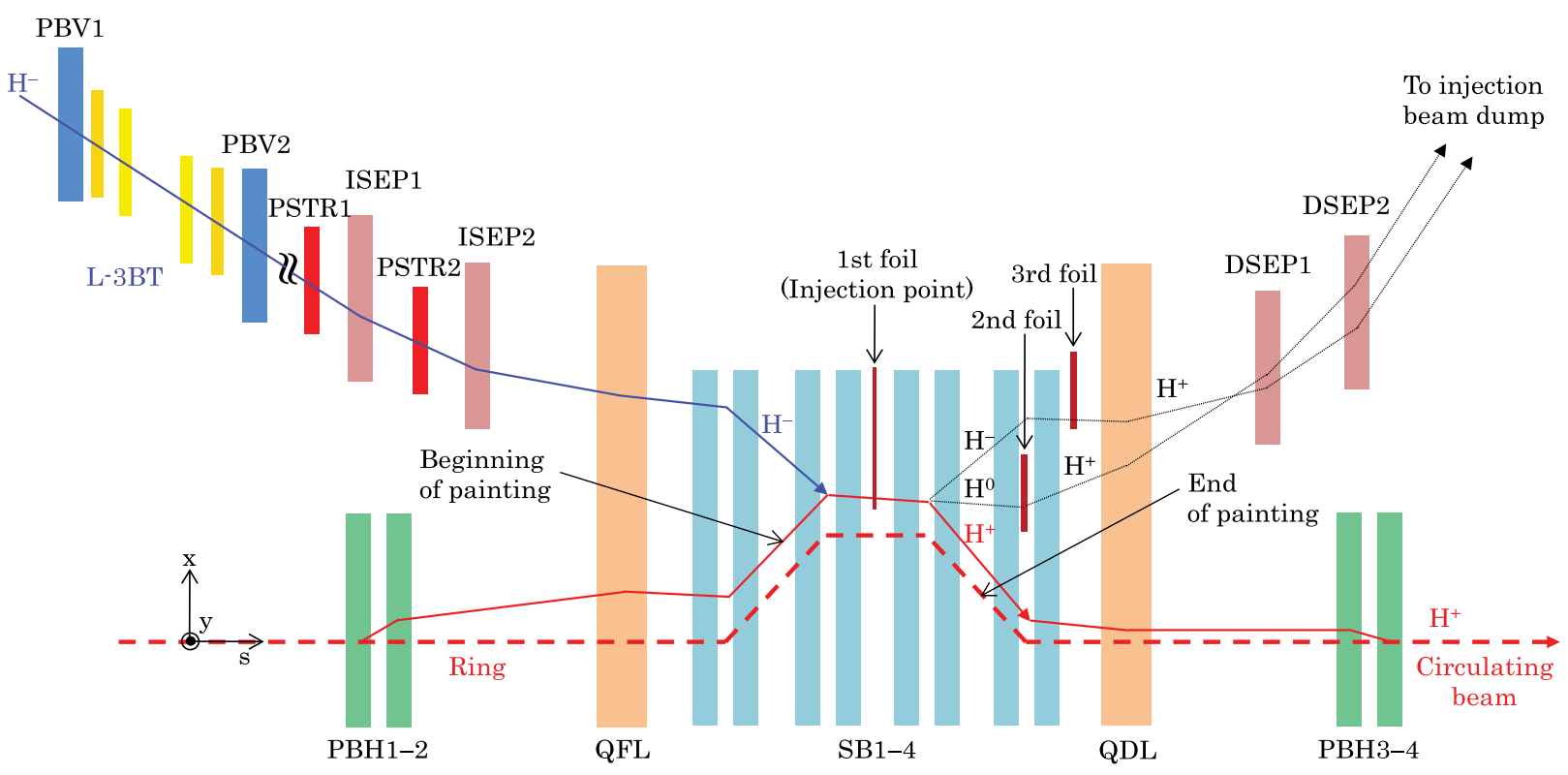

FIG. 4. Schematic view of the RCS injection area and demonstration of a transverse painting injection. In the horizontal direction, injected beam trajectory (blue line) is matched and fixed to the circulating orbit bump offset (red line) produced by the SB1-4 and PBH1-4 as indicated by "Beginning of painting". The injection painting is performed through sweeping circulating orbit bump offset by the PBH1-4 during $0.5 \mathrm{~ms}$ injection period to the red dashed line indicated by "End of painting". The SB1-4 is then linearly ramped down to zero so the closed orbit is moved to the ring center. However, for the center (no painting) injection, the red broken line is the orbit bump offset produced by the SB1-4. The injected beam trajectory as well as the first foil are then accordingly moved. There is no close orbit variation during injection as thus no transverse painting. 
( $\mathrm{x}$ and $\mathrm{x}^{\prime}$ ) of the injected beam center is matched at a bumped closed orbit made by four chicane bump magnets, SB1-4 together with four horizontal painting bump magnets, PBH1-4. The horizontal phase space painting is performed by sweeping the closed orbit by the PBH1-4 during the injection period of $0.5 \mathrm{~ms}[4,6]$. After the injection is finished, SB1-4 is linearly ramped down to zero so as to move the closed orbit to the ring center. In the vertical direction, however, vertical angle $\left(\mathrm{y}^{\prime}\right)$ of the injected beam at the foil is directly controlled pulse to pulse by two vertical painting bump magnets, PBV1-2 placed in the L-3BT line. They are located almost $180^{\circ}$ upstream betatron phase advance from the stripper foil.

The black and red ellipses in Fig. 5 represent painting emittance for MLF and MR, respectively. The phase space
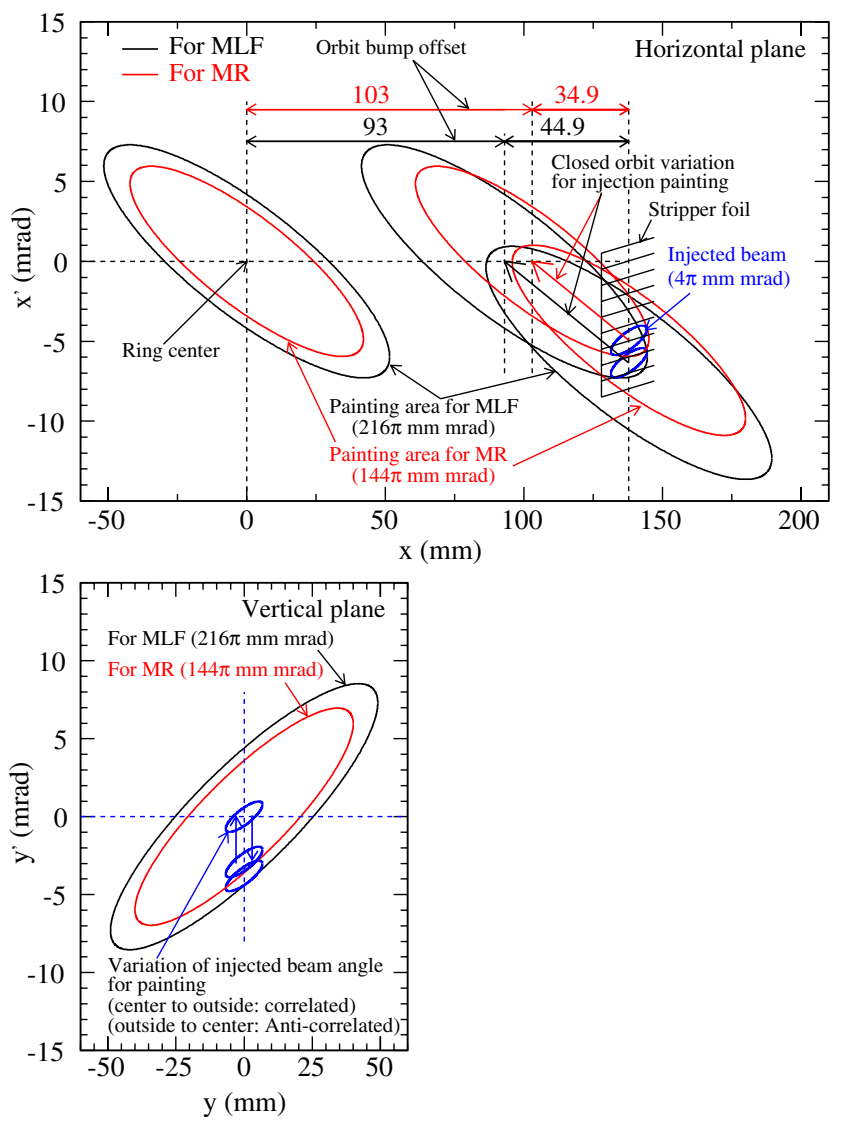

FIG. 5. Schematic view of the transverse injection painting process in the RCS for horizontal and vertical planes as shown in the top and bottom figures, respectively. A closed orbit variation is done in the horizontal plane, while the injected beam angle itself is changed in the vertical direction. The design painting emittance for the MLF and MR are 216 and $144 \pi \mathrm{mm} \mathrm{mrad}$, respectively. As the stripper foil position is fixed, the only choice for switching a smaller painting area for the MR has to be done by changing an angle of the injected beam. The power supplies of the ISEP1-2 are dc and thus PSTR magnets are additionally installed for that purpose. The PBV1-2 located in the injection BT line are used for directly changing vertical angle of the injected beam to the change vertical paining area pulse to pulse. coordinates of the injected beam shown by blue colors determine the painting area. For a designed painting area of $216 \pi \mathrm{mm} \mathrm{mrad}$ for the MLF, the SBs produce a flat horizontal orbit bump offset of $93 \mathrm{~mm}$, where phase space coordinate offset produced by PBHs is $44.9 \mathrm{~mm}$ and $-6.3 \mathrm{mrad}$. The $\mathrm{x}$ and $\mathrm{x}^{\prime}$ of the injected beam centroid are thus $137.9(93+44.9) \mathrm{mm}$ and $-6.3 \mathrm{mrad}$, respectively, where the foil is placed (see also Fig. 4). In the same manner, phase space coordinate offset produced by the PBHs for a painting area of $144 \pi \mathrm{mm}$ mrad for the MR should be $34.9 \mathrm{~mm}$ and $-4.9 \mathrm{mrad}$. The $\mathrm{x}$ and $\mathrm{x}^{\prime}$ of the injected beam are thus $127.9(93+34.9) \mathrm{mm}$ and $-4.9 \mathrm{mrad}$, respectively. In principle, one can thus realize the goal by changing injected and circulating beam orbits in a pulse-to-pulse mode. However, there remains an issue to be cleared. As the foil position cannot be changed pulse to pulse, it has to move $10 \mathrm{~mm}$ further inside in the horizontal direction in order to cover injection beam for the MR painting. The injected beam center from the foil edge is usually kept $8 \mathrm{~mm}$ for the operation. As a result, the centroid of the injected beam from the foil edge would be then as much as $18 \mathrm{~mm}$ for the MLF painting. The uncontrolled beam loss due to the foil scattering of the circulating beam is thus severely increased for the MLF painting. Similar to any other accelerator using a similar injection scheme, such an uncontrolled beam loss at the injection area is a big concern also in the $3-\mathrm{GeV}$ RCS of J-PARC. It is one of the most uncontrolled beam loss sources in the RCS and already a serious issue even at the present operation with a beam power of $300 \mathrm{~kW}$ [8,9].

In order to keep control of the foil scattering beam loss, we proposed an efficient way for switching MLF painting area to the MR one by changing only the angle of the injected beam to a smaller value (see Fig. 5). That means an injected beam angle of $-6.3 \mathrm{mrad}$ for the MLF is changed to $-4.9 \mathrm{mrad}$ for the MR but the beam position is kept unchanged at $137.9 \mathrm{~mm}$. It has to be mentioned that the orbit bump offset by the SBs should be then about $11 \%$ higher and has to be changed for a height of $103 \mathrm{~mm}$ for the MR as compared to $93 \mathrm{~mm}$ for the MLF. The $\mathrm{x}$ and $\mathrm{x}^{\prime}$ of the injected beam are thus $137.9(103+34.9) \mathrm{mm}$ and $-4.9 \mathrm{mrad}$, respectively. As power supplies of both ISEP1 and ISEP2 are dc, two PSTR magnets are thus designed and installed for that purpose. The horizontal injected beam orbit for the MLF painting is fixed by ISEP1-2. The PSTR magnets for the MR painting are used in addition to the already fixed ISEP1-2 in order to change for a required smaller angle of the injected beam at the foil by keeping its position unchanged as fixed for the MLF painting.

A pulse-to-pulse switching of the vertical painting area MLF to MR, however, is done by controlling the vertical angle of the injected beam at the stripper foil directly by the vertical painting magnets, PBV1-2 as they are located in the L-3BT line. The vertical angle of the injected beam 
in the circulating phase space can be sweep either center to outside or outside to center for so-called correlated or anticorrelated painting.

The PSTR magnets are designed for two purposes in the $400 \mathrm{MeV}$ injection. First, they will be used for switching a relatively larger MLF painting area to that of a smaller one for the MR as discussed above. Second, they will also be necessary for no painting or so-called center injection normally done in beam studies and basic parameter optimization. In this case injected beam orbit is merged with constant circulating orbit bump offset produced by only the chicane bump magnets, SB1-4. There is no closed orbit variation during injection and thus no transverse painting. At the present $181 \mathrm{MeV}$ injection energy, injected beam trajectory for the center injection is adjusted by using ISEP1-2. However, the power supply of the injection septa, especially ISEP2 exceeds the capacity in order to realize such a center injection for the $400 \mathrm{MeV}$ injected beam. We considered completely separate power supplies for each individual purpose because of the following reason. The magnetic fields of PSTRs required for changing painting area MLF to MR are considerably low and nearly 1 order of magnitude lower as compared to those needed for the center injection. In order to make sure for good performances, power supplies of the PSTR magnets are separated for each purpose. For changing the painting area, there are two independent and bipolar pulsed power supplies capable of a maximum current of $\pm 450 \mathrm{~A}$, which gives a maximum magnetic field of $\pm 0.026 \mathrm{~T} \mathrm{~m}$. On the other hand, two independent and bipolar but dc power supplies with a maximum current of $\pm 3000 \mathrm{~A}$ are prepared for the center injection. In this case the maximum magnetic field is $\pm 0.174 \mathrm{~T} \mathrm{~m}$ [10-12]. The choice of using ac or dc power supply can be switched by a remote control program.

In order to fix final specifications of the PSTR in the design stage, we took into account all possible scenarios for the $400 \mathrm{MeV}$ injection. All possible betatron tunes set for the RCS in the range of 6 to 7 for both horizontal and vertical directions are considered. The latest tune diagram of the RCS obtained for a single particle dynamic aperture calculation has been reported in Ref. [13]. The betatron tunes are changed by changing focusing strength of the ring quadrupoles and thus Twiss parameters of the circulating beam at the stripper foil is changed. The shape of the bigger ellipses shown in Fig. 5 is changed, resulting a change of the phase space coordinates of the injection point as for a desired painting area, injected beam has to be set at the right edge position of the phase space of the circulating beam. In the horizontal direction, injection phase space coordinates $\mathrm{x}$ and $\mathrm{x}^{\prime}$ for a painting area of $216 \pi \mathrm{mmmrad}$ is changed as much as $\pm 6 \mathrm{~mm}$ and $\pm 1.3 \mathrm{mrad}$, respectively, due to betatron tune variation in the range of 6-7.

It is important to mention that injected as well as circulating beam orbits pass off center of the injection quadrupole magnet named QFL and thus both orbits are changed for a different focusing strength of the QFL. This is because the deflection angle for an off center beam passing through a quadrupole magnet is generally taken into account as a product of the focusing strength of the quadrupole and the displacement of the orbit from the quadrupole center. The QFL is located just upstream of the first chicane bump magnets, SB1 (see Fig. 3). The injected beam trajectory passes very off center and more than $0.2 \mathrm{~m}$ from the QFL center, while circulating bumped orbit at the beginning for a $216 \pi \mathrm{mm}$ mrad painting injection passes about $0.06 \mathrm{~m}$ from the QFL center. For a nominal operating tune, where QFL focusing strength is $0.2 \mathrm{~m}^{-1}$, the deflection angle acts on the injected beam trajectory passing $0.2 \mathrm{~m}$ off center is thus $40 \mathrm{mrad}$ and it proportionally changes for a change in quadrupole strength, resulting in a change of the injected beam orbit at the downstream of the QFL. The injected beam trajectory should be merged with the circulating closed orbit at the stripper foil location and thus any change of the QFL should be covered by the ISEPs for the MLF painting and PSTRs in addition for switching painting area for the MR. We have also considered a little more variation of switching the painting area down to $100 \pi \mathrm{mm}$ mrad for the MR, although it is originally $144 \pi \mathrm{mm} \mathrm{mrad}$.

Figure 6 shows required magnetic fields of the PSTR magnets in order to realize a painting area down to $100 \pi \mathrm{mm}$ mrad for the MR beam switched from the MLF painting area of $216 \pi \mathrm{mm}$ mrad based on the parameter survey for all possible scenarios. The box in red color

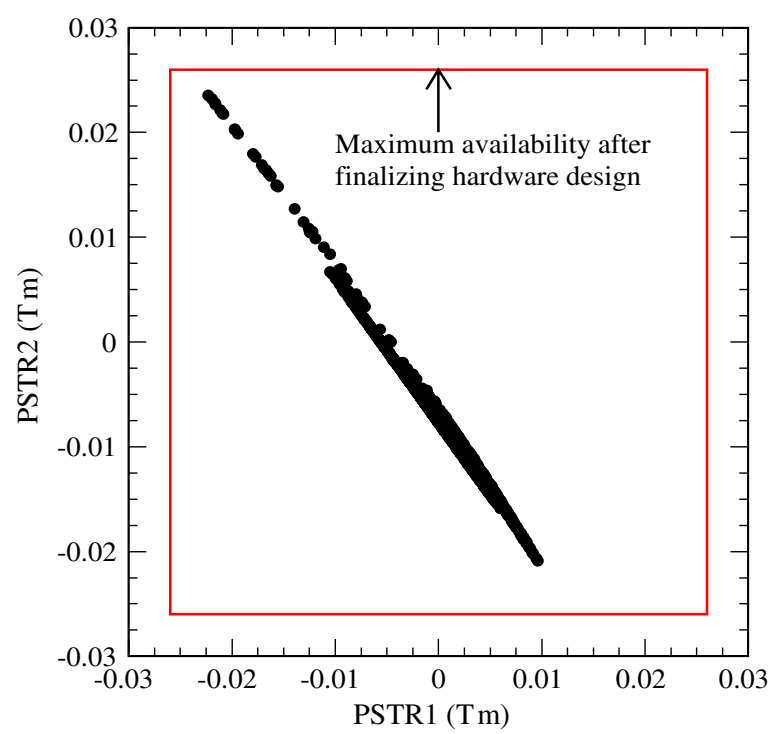

FIG. 6. Required magnetic fields of the PSTR magnets for a painting area of $100 \pi \mathrm{mm}$ mrad for the MR switching from a MLF painting area of $216 \pi \mathrm{mm} \mathrm{mrad}$ based on the survey for all possible scenarios at the $400 \mathrm{MeV}$ injection. The red box shows the maximum availability of final hardware designing based on the magnetic field measurement of the magnets. The corresponding maximum current for the pulse mode power supply is \pm 450 A. 
shows the maximum availability of final hardware designing and based on the magnetic field measurement.

It is worth mentioning that a fluctuation of the ac power supply operating for several hours with $400 \mathrm{~A}$ is measured by a current transformer (CT). The corresponding maximum fluctuation of the magnetic field is then calculated to be negligibly small as $\pm 0.1 \%$. Consequently, injected beam coordinates $\mathrm{x}$ and $\mathrm{x}^{\prime}$ at the stripper foil location are estimated to fluctuate by a maximum of $\pm 0.02 \mathrm{~mm}$ and $\pm 0.002 \mathrm{mrad}$, respectively, and thus the effect is considered to be negligibly small.

At this stage the numerical simulations for determining overall specifications of the PSTR magnets were performed based on the single particle beam dynamics by using the SAD simulation tool [14].

\section{EXPERIMENTAL RESULTS AND COMPARISON WITH NUMERICAL SIMULATIONS}

The PSTR magnets were installed in the 2012 summer shutdown period and afterwords they were commissioned with beam in late November. A detail field measurement was performed before installation. A detail of the hardware designing as well as results of the field measurement have already been presented elsewhere [11,12]. Based on the field measurement data, a rather linear relation between the input current and the magnetic field is obtained. In the simulation, a required bending angle of each PSTR magnet is determined, which is then transformed to the magnetic field multiplying by the magnetic rigidity of the injected beam energy. The required current from the power supply for the corresponding magnetic field is supplied to the magnet for appropriate excitation. The experimental studies and the corresponding results obtained with PSTR magnets are categorized for the following four topics: (i) switching MLF painting area to the MR and a comparison with designed parameters; (ii) confirmation of a $400 \mathrm{MeV}$ injection energy equivalent center (no painting) injected orbit with dc power supplies of the PSTR magnets; (iii) performance of the PSTR magnets for changing painting area MLF to MR in a pulse-to-pulse mode for one cycle trial user operation of the RCS; and (iv) measurement of the extracted beam profiles and confirmation of the designed principle for ensuring a smaller emittance for the MR as compared to the MLF.

\section{A. Switching painting area MLF to MR}

By using PSTR magnets we first carried out an experimental test for switching MLF painting area of $150 \pi \mathrm{mmmrad}$ to the MR painting area of $100 \pi \mathrm{mm}$ mrad. It is important to mention that at present a maximum painting area within an acceptable beam loss is unfortunately limited up to about $150 \pi \mathrm{mm} \mathrm{mrad}$ due to mainly a large vertical beta beating (as much as more than $30 \%$ around the ring) caused by the edge effect of the chicane bump magnets during beam injection and also for some other lattice imperfections $[13,15]$. These lead to a significant reduction of the dynamic aperture of the ring but at present there exist no knobs for compensating these effects. However, a total of six additional quadrupole magnets have already been designed for correcting those effects and will be installed in $2013[15,16]$. The transverse painting area can be then expanded to the designed one of $216 \pi \mathrm{mm}$ mrad.

Figure 7 shows the horizontal injected beam trajectory from upstream of the ISEP1 to the stripper foil. The black and red lines are calculated for expected injected beam trajectories for the MLF $(150 \pi \mathrm{mm} \mathrm{mrad})$ and the MR $(100 \pi \mathrm{mm} \mathrm{mrad})$ painting area, respectively. The corresponding measured data at four locations are depicted by the open black and solid red circles, respectively. The injected beam position was measured by three multiwire profile monitors (MWPM2-4) and a beam position monitor named IBPM (see Fig. 3). The MWPM gives both centroid position as well as width of the injected beam, while IBPM gives only the centroid position of the beam.

As described earlier, the injected beam trajectory for the MLF painting is fixed by the ISEPs, while in addition PSTRs are used for the MR painting in order to change injected beam angle to a desired smaller value at the stripper foil by keeping the position the same as that for

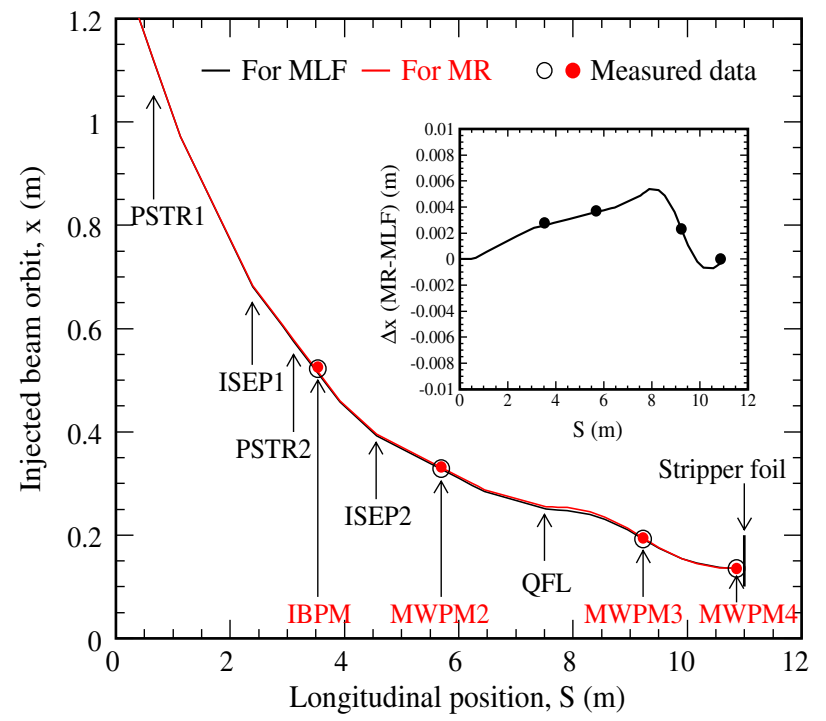

FIG. 7. A comparison of the calculated (black and red lines) and measured (open and solid circles) injected beam orbits for the MLF (150 $\pi \mathrm{mm} \mathrm{mrad)} \mathrm{and} \mathrm{MR}(100 \pi \mathrm{mm} \mathrm{mrad})$ painting, respectively. The horizontal axis is the longitudinal position along the RCS central orbit, where the vertical axis is the horizontal position of the injected beam with respect to the RCS central orbit. The difference of the two orbits is shown in the inset. The measured beam position for both MLF and MR painting orbits as well as the difference of the two orbits are found to be quite consistent with the expected ones produced by the numerical simulation. 
MLF. The measured beam positions are found to be quite consistent with the numerical simulations. For a further detailed comparison, a difference of the two trajectories is shown in the inset and thus a consistency in between simulation and measurement can easily be justified. The maximum difference of the two trajectories is about $6 \mathrm{~mm}$ near the QFL. The bending angles of the PSTR1 and PSTR2 determined in the simulations are only -0.9 and 0.42 mrads, respectively. The corresponding magnetic fields are calculated to be $-1.83 \times 10^{-3}$ and $0.85 \times$ $10^{-3} \mathrm{Tm}$, respectively, for which the ac currents supplied to the magnets are 33 and $-15 \mathrm{~A}$, respectively. It may be interesting to mention that magnetic fields of the ISEP1 and ISEP2 are more than 2 order of magnitude higher than those of PSTRs and are -0.332 and $-0.197 \mathrm{~T} \mathrm{~m}$, respectively. It may thus show that such a level of controlling the injected beam trajectory by using the PSTR magnets can be well predicted in the numerical simulation as it has been confirmed in the measurement. The reliability of the PSTR magnets for the present purpose can also be justified well.

\section{B. Confirmation of a $400 \mathrm{MeV}$ injection energy equivalent center (no painting) injected orbit}

As also mentioned earlier, one of the other purposes with PSTR magnets is to perform the no painting or the so-called center injection. In this case, injected beam has to be merged with circulating orbit bump offset produced by only the chicane bump magnets. The orbit bump offset at the stripper foil location is $93 \mathrm{~mm}$ and is time independent during the injection period. At the present $181 \mathrm{MeV}$ injection energy, injected beam trajectory for the center injection can be adjusted by the ISEP1-2 but for the $400 \mathrm{MeV}$ injection, power supply of the ISEP2 will exceed the capacity and thus PSTR1-2 will be used in addition to the ISEP1-2.

Figure 8 shows the expected injected beam trajectories (lines) and measured beam positions (symbols) at four beam monitor locations for the center injection. The calculated injected beam trajectory and the measured data for the present $181 \mathrm{MeV}$ injection energy are shown by the black line and black open circles, respectively. The red lines and the red solid circles are those for equivalent center injection trajectories for the $400 \mathrm{MeV}$. The ISEP1-2 are only used for the $181 \mathrm{MeV}$, while PSTR1-2 are additionally used for an equivalent center injection trajectory for the $400 \mathrm{MeV}$. The bending angles of ISEPs, especially for ISEP2, were fixed for a certain value, nearly maximum of its capacity for the $400 \mathrm{MeV}$ injected beam, and then PSTR1-2 are additionally used in order to produce an equivalent center injected orbit for that energy. Similar to the case of switching painting area by the PSTRs (see Fig. 7), here also the measured $400 \mathrm{MeV}$ equivalent center injection beam trajectory is found to be consistent with the expected one. The red line will be the reference center injection orbit for the $400 \mathrm{MeV}$ injected beam. An expected difference of the two center injected orbits (181 MeV and $400 \mathrm{MeV}$ ) shows a good

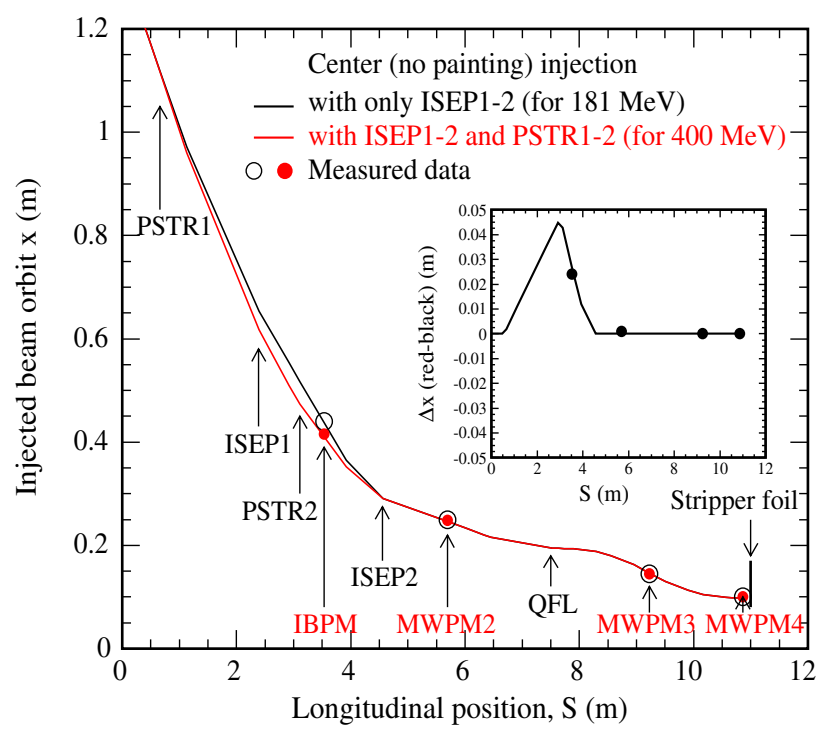

FIG. 8. Calculated injected beam trajectories (lines) and measured beam positions (symbols) at four locations are shown for the center injection. The black line and the black open circles are for the present $181 \mathrm{MeV}$ injected beam, while red lines and red solid circles are for an equivalent center injection trajectory for the $400 \mathrm{MeV}$ injected beam. ISEP1-2 are only used for the $181 \mathrm{MeV}$ while PSTR1-2 in addition to the ISEP1-2 are used for the equivalent center injection orbit at the $400 \mathrm{MeV}$. A difference of the two orbits is shown in the inset. A good consistency between expected and measured data has also been demonstrated here.

agreement with measured data as shown in the inset. A detailed parameter list of the PSTR1-2 including ISEP1-2 is given in Table I.

\section{Performance of the PSTR magnets for one cycle trial user operation of the RCS}

Although PSTR magnets will be put in real operation for the upgraded $400 \mathrm{MeV}$ injection energy in 2014, we have

TABLE I. A summary of required currents of the ISEPs and PSTRs power supplies for center injection in the present $181 \mathrm{MeV}$ and upgraded $400 \mathrm{MeV}$ injected energies. The measured $181 \mathrm{MeV}$ injection parameters are scaled for an increase of the magnetic rigidity $(\mathrm{B} \rho)$ in order to obtain those required for the $400 \mathrm{MeV}$. The capacity of ISEP2 for the center injection at $400 \mathrm{MeV}$ exceeds the limit and thus PSTRs are needed in addition to ISEPs. The reference center injection orbit obtained by using PSTRs and ISEPs at the present $181 \mathrm{MeV}$ has already been confirmed that such a center injection orbit can thus be achieved at the $400 \mathrm{MeV}$ as currents for all power supplies are calculated to be within the capacity.

\begin{tabular}{lcccc}
\hline \hline Energy (MeV) & ISEP1 (A) & ISEP2 (A) & PSTR1 (A) & PSTR2 (A) \\
\hline 181 & 1184 & 5325 & 0 & 0 \\
400 & 1852 & $8328(\times)$ & 0 & 0 \\
181 & 1184 & 3994 & -602 & 1878 \\
400 & 1852 & 6247 & -942 & 2937 \\
\hline \hline
\end{tabular}



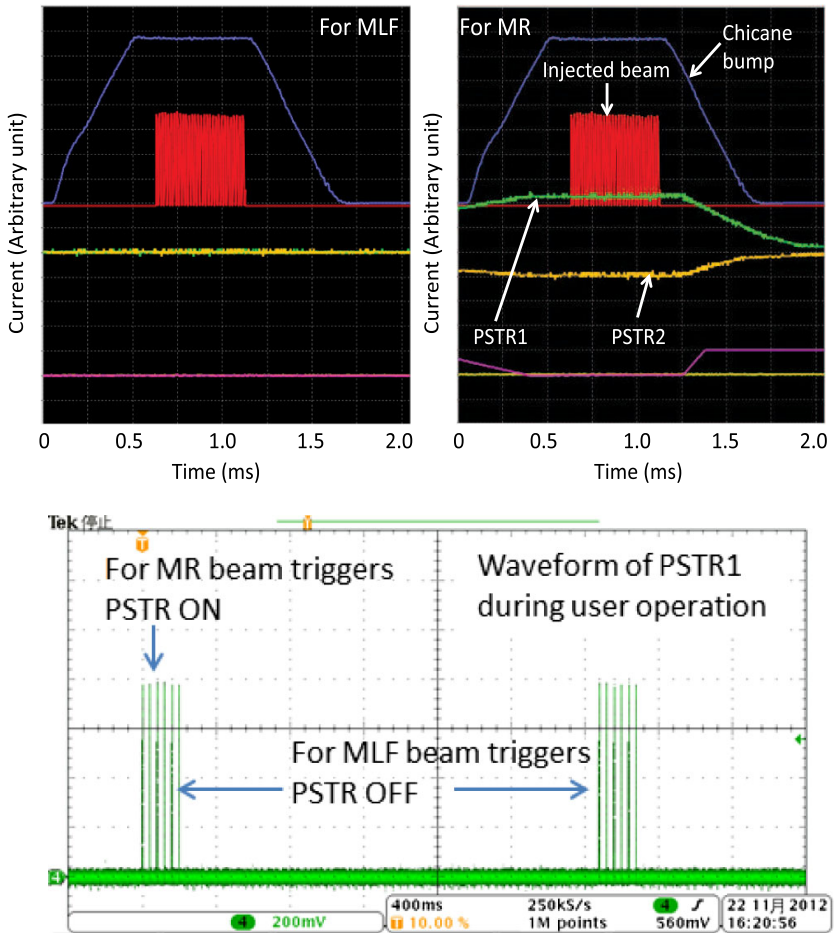

FIG. 9. Waveform of the PSTR magnets together with chicane bump magnet while in operation. They were found to be working as designed.

already checked their performances in advance for one cycle trial operation of the RCS in the present $181 \mathrm{MeV}$ injection with a beam power of $300 \mathrm{~kW}$ at $25 \mathrm{~Hz}$. A correlated transverse painting area of 150 and $50 \pi \mathrm{mm} \mathrm{mrad}$ for the horizontal and vertical direction, respectively, set for the MLF, is switched to a painting area of $100 \pi \mathrm{mm} \mathrm{mrad}$ for both directions for the MR. As the procedures explained earlier, the horizontal injected beam trajectory painting for the MLF was determined by the ISEP1 and ISEP2. They were fixed and PSTR1-2 are additionally used for the MR in order to change for a required smaller angle of the injected beam at the stripper foil but keeping its position unchanged from that of MLF. The PBV1-2 are programmed in order to control the vertical angle of the injected beam at the foil for each painting area. The horizontal injection painting magnets, $\mathrm{PBHs}$, are also used for each corresponding painting parameter for the MLF and MR, respectively.

Figure 9 (top) shows an online snapshot of the PSTR waveforms and the chicane bump coincided with injected beam (red). As programmed, they were ON only for the MR beam trigger as depicted by the green and yellow lines for the PSTR1 and PSTR2, respectively. A further separate snapshot of a PSTR waveform for a longer time range is also shown in the bottom figure. The MR operation cycle was $2.48 \mathrm{~s}$ and thus in every $2.48 \mathrm{~s}$, PSTRs were ON for the 6 MR beam triggers, while they were OFF for the rest of the time allocated for the MLF beam triggers. The first four

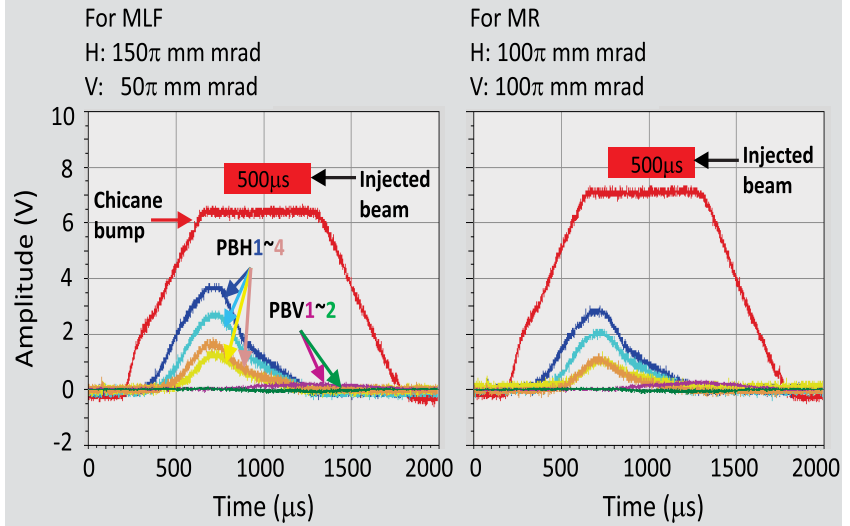

FIG. 10. Waveforms of painting and chicane bump magnets for the MLF (left) and MR (right) painting. The painting areas for the MLF was 150 and $50 \pi \mathrm{mm}$ mrads for the horizontal and vertical direction, respectively, where it was $100 \pi \mathrm{mm} \mathrm{mrad}$ for both directions for the MR.

pulses are injected to the MR while the last two pulses are with no beam triggers in order to make sure there was no effect of the eddy current for the MLF beam from the beam switching pulse bending magnet (PB) located downstream of the RCS extraction beam transport line (see Fig. 1).

Figure 10 shows typical waveform (WF) of the horizontal and vertical painting magnets (PBH1-4 and PBV1-2) together with the chicane bump magnets. The left figure is for the MLF painting where the right one is that for the MR. The height of each WF changed for respective painting area can be seen. It can also be confirmed that the chicane bump height for the MR was also accordingly changed and was about $10 \%$ higher as compared to the MLF painting due to the principle explained in Sec. II and also as shown in Fig. 5.

Figure 11 shows an online trend of the horizontal injected beam position measured by the IBPM (see Fig. 3) while PSTRs were in operation for switching the MLF painting area to the MR one. The beam position is given as a measured distance of the beam centroid from the beam duct center at the IBPM. The data shown in black color is for the MLF beam triggers, where the red ones are those for the MR. The expected injected beam position at the IBPM for the MR painting is about $3 \mathrm{~mm}$ further on the outer side of beam pipe as compared to that for the MLF painting, which is successfully confirmed in the measurement. The data was taken for about an hour, while for the former half beam was delivered to the MR mostly by on demand single shot. There was thus only a few countable shots in the MR for that period. The MR user operation was started at the end of the day and more shots were thus recorded then. As expected, the measured beam position for the MR was also found to be very stable. It then reflects a good stability of the PSTR power supplies even for such low operating currents and eventually guarantees a well controlled painting area. 


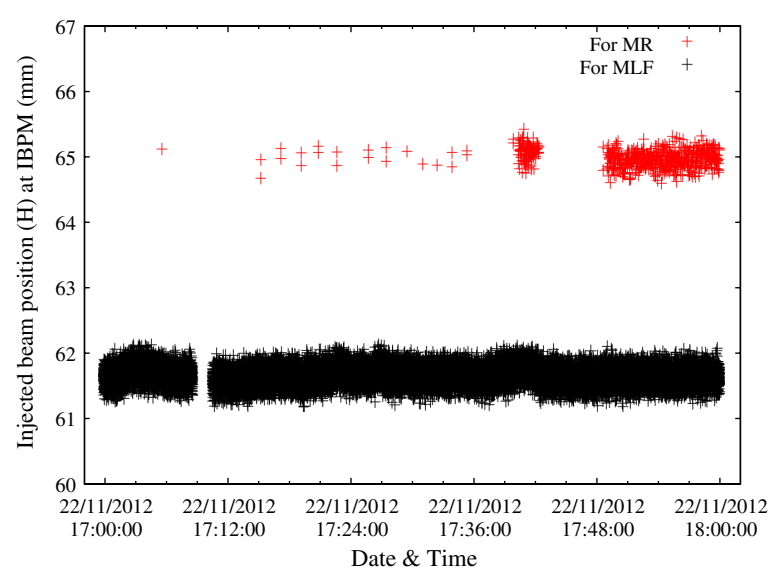

FIG. 11. An online trend of the horizontal injected beam position measured by the IBPM (see Fig. 3) for MLF (PSTR off) and MR (PSTR on) beam triggers during user operation of the RCS. The data taking period was about $1 \mathrm{~h}$, while for the former half beam was delivered to the MR mostly by on demand single shot. The expected relative difference of the beam position is about $3 \mathrm{~mm}$ and was thus consistent with expectation. A good stability of the measured beam position for the MR reflects a good stability of the PSTR power supplies and eventually guarantees good control of the painting area.

The main goal of the newly designed PSTR magnets for switching transverse painting area in a pulse-to-pulse mode for the RCS operation is thus confirmed well in advance before they will be put in operation for the upgraded $400 \mathrm{MeV}$ injection in 2014.

\section{Measurement of the extracted beam profiles for the MLF and MR painting}

In order to experimentally demonstrate controlling of the extracted beam emittance through injection painting, we have measured extracted beam profiles for the MLF and MR painting. For simplicity, transverse injection painting was applied only for the horizontal direction and was 150 and $100 \pi \mathrm{mm}$ mrad for the MLF and MR, respectively. The extracted beam profile width for the MR painting was thus expected to be narrower as compared to that of MLF painting. The extracted beam profiles were measured by an MWPM placed in the beam transport line of 3-GeV RCS to the neutron target (3-NBT).

In this experiment, the peak current of the linac beam was $17 \mathrm{~mA}$ and the studies were performed for two beam intensities. The beam intensity was controlled by the chopper beam-on duty factor and was $56 \%$ and $26 \%$ corresponding to $2.91 \times 10^{13}$ and $1.25 \times 10^{13} \mathrm{ppp}$, respectively. The extracted beam power at the $3 \mathrm{GeV}$ was 350 and $150 \mathrm{~kW}$, respectively, if RCS was running at a repetition rate of $25 \mathrm{~Hz}$. In order to reduce emittance growth due to the space charge effect at the lower energy region, the beam intensity was reduced to more than a half for the later case. The measurement was done by on demand single shot beam.
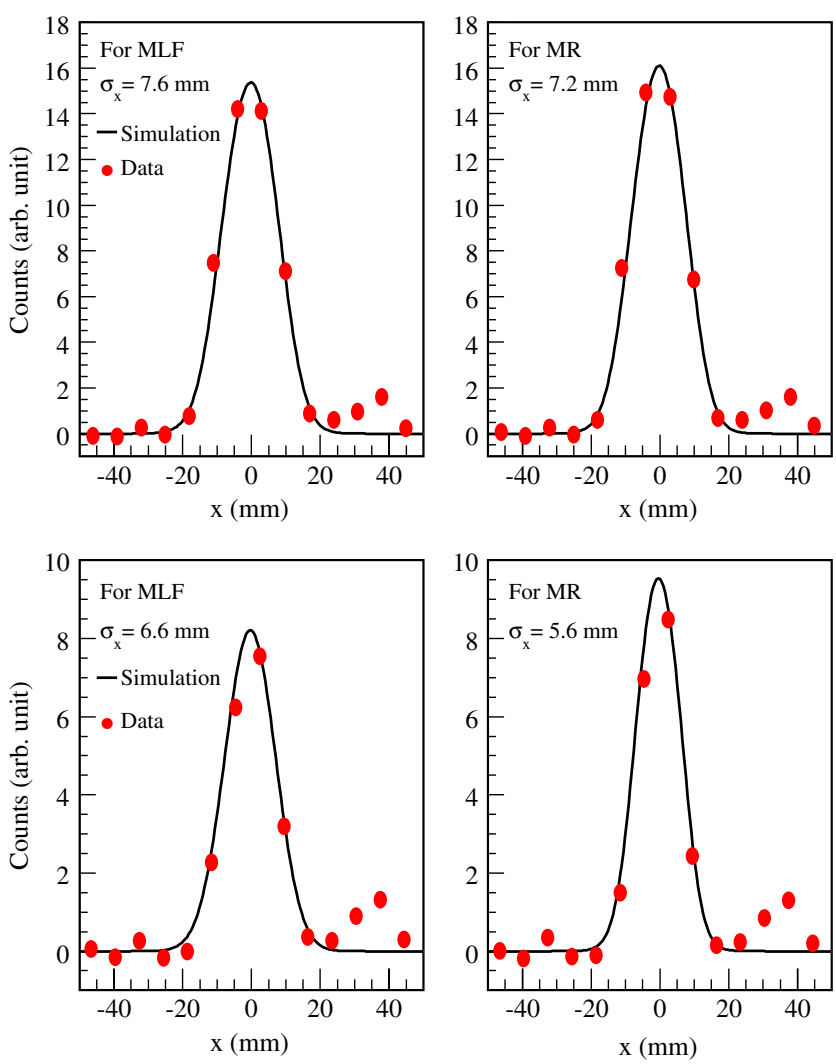

FIG. 12. Comparison of the extracted beam profiles for the MLF and MR painting. The upper plots are for a beam power of $350 \mathrm{~kW}$, where the lower ones are those for $150 \mathrm{~kW}$. The measured beam profile by the MWPM are depicted by red solid circles, where lines are the corresponding numerical simulation. The profile width with an initial smaller injection painting area for the MR (right) is confirmed to be narrower as compared to that of a larger painting area for the MLF (left). The difference is more significant for a relatively lower beam intensity as can be seen in the lower plots. The corresponding simulation results are found to be quite consistent to those with measurements.

We have also performed corresponding numerical beam simulations by using ORBIT 3D particle tracking code. The realistic machine parameters were introduced in the simulation in the same manner as was in the present experiment. It is worth mentioning that the ORBIT code has recently been given new advances and capabilities for realistic beam simulations in synchrotrons $[17,18]$. By introducing all relevant machine parameters, a relatively good model has been constructed for beam simulation in the $3-\mathrm{GeV}$ RCS of J-PARC. The first simulation result for a relatively high intensity beam of more than $400 \mathrm{~kW}$ has found to reproduce the experimental data well [18].

Figure 12 shows measured horizontal extracted beam profiles compared with simulated ones for the two intensities of $2.91 \times 10^{13} \mathrm{ppp}$ (upper plots) and $1.25 \times$ $10^{13} \mathrm{ppp}$ (lower plots), respectively. The left figures are 
TABLE II. A list of the beam parameters and the corresponding measured and simulated horizontal rms width of the extracted beam at $3 \mathrm{GeV}$. The studies were done for two beam intensities. One is for a present maximum beam power of $350 \mathrm{~kW}$, while the other one was for a comparatively lower beam power of $150 \mathrm{~kW}$ at the extraction energy. The painting area for the MLF and MR was 150 and $100 \pi \mathrm{mm}$ mrad, respectively. As expected, extracted beam profiles for the MR are measured to be narrower as compared to those for the MLF. The difference is more significant for a comparatively low intensity beam. The corresponding quantitative simulation results are also found to be consistent with measured data.

\begin{tabular}{lccccc}
\hline \hline For & $\begin{array}{c}\text { Painting } \\
(\pi \mathrm{mm} \text { mrad })\end{array}$ & $\begin{array}{c}\text { Beam } \\
\text { intensity }(\mathrm{ppp})\end{array}$ & $\begin{array}{c}\text { Equivalent beam } \\
\text { power }(\mathrm{kW})\end{array}$ & $\begin{array}{c}\sigma_{x}(\text { Measured }) \\
(\mathrm{mm})\end{array}$ & $\begin{array}{c}\sigma_{x}(\text { simulated }) \\
(\mathrm{mm})\end{array}$ \\
\hline MLF & 150 & $2.91 \times 10^{13}$ & 350 & $7.6 \pm 0.1$ & 7.4 \\
MR & 100 & $2.91 \times 10^{13}$ & 350 & $7.2 \pm 0.1$ & 7.1 \\
MLF & 150 & $1.25 \times 10^{13}$ & 150 & $6.6 \pm 0.2$ & 6.6 \\
MR & 100 & $1.25 \times 10^{13}$ & 150 & $5.6 \pm 0.2$ & 5.7 \\
\hline \hline
\end{tabular}

for the MLF beam, while the right ones are for the MR beam. The solid red circles represent the measured beam profiles, where lines are the corresponding simulated beam profiles. The measured beam profiles for both intensities are found to be well reproduced by the corresponding simulation. As expected, the width of the extracted beam profile for the MR painting is measured to be narrower as compared to that of MLF painting. The difference of the profile widths between MLF and MR beam is more significant, especially for a low intensity beam shown in the lower plots. This is mainly because the emittance growth due to the space charge effect in the lower energy region and subsequent effect of the nonlinear field component as well as lattice imperfections are much suppressed for such an intensity beam. A detailed list of the beam parameters and the corresponding measured and simulated rms widths of the extracted beam profiles are given in Table II.

The present result shows direct evidence that the transverse emittance of the extracted beam can be controlled through optimizing initial phase space injection painting as requested by the users even in the simultaneous operation. The difference of the two profiles would be more significant when a design MLF painting area of $216 \pi \mathrm{mm}$ mrad can be switched to the MR designed painting area of $144 \pi \mathrm{mm}$ mrad as demonstrated in Fig. 2. At present there exists a limitation on expanding a transverse injection painting area larger than $150 \pi \mathrm{mm}$ mrad. This is mainly because of a large vertical beta beating around the ring (as much as more than 30\%) caused by the edge effect of the chicane bump magnets during beam injection and also for some other lattice imperfections [13,15]. The dynamic aperture of the RCS is then greatly reduced and consequently leads to an unacceptable beam loss but there exist no knobs for compensating these effects at present. However, a total of six quadrupole corrector magnets for dynamic correction of these effects have already been designed and will be installed in the ring in $2013[15,16]$.
The transverse injection painting area can be then expanded to the designed one of $216 \pi \mathrm{mm}$ mrad.

\section{SUMMARY}

In order to ensure desired extracted beam emittances in a multiuser high intensity proton synchrotron, a pulse-topulse direct control of the transverse injection painting area is proposed and also been verified through experimental studies in the 3-GeV rapid cycling synchrotron of the Japan Proton Accelerator Research Complex. For that purpose, two horizontal pulse steering magnets are designed and installed in the injection beam transport line. Their main purpose is to change a pulse to pulse of a bigger transverse painting area to a smaller one in simultaneous operation of the RCS for two downstream facilities. They will also be definitely needed for the center (no painting) injection for the upgraded $400 \mathrm{MeV}$ injection energy to that of present $181 \mathrm{MeV}$ due to limitation of the second injection septum. The beam commissioning of the PSTR magnets was first done in 2012 autumn beam studies and they were proved to be functioned as designed for both purposes. A switching of a larger MLF painting area to smaller one for the MR has also been successfully carried out for one cycle trial operation of the RCS. A control of the extracted beam emittance through well optimizing transverse injection painting area has also been experimentally demonstrated for two beam intensities. The experimental results are also found to be quite consistent with those obtained in the numerical simulations.

It is thus confirmed that in a multiuser machine beam parameters can be dynamically controlled and delivered as requested by the users even in simultaneous operation. A successful experimental demonstration of the present principle to control extracted beam emittances in simultaneous operation is obviously a remarkable progress in the recent high intensity accelerator in order to meet user requests in such an advanced way. The present principle is very straightforward and can be applicable to any similar 
multiuser machine for controlling beam emittances in a pulse-to-pulse mode.

\section{ACKNOWLEDGMENTS}

The authors highly acknowledge all members of the RCS; namely, $400 \mathrm{MeV}$ task force members and control group members for numerous support, discussions, and cooperation during design stage, installation, field measurement, designing real time operation patterns, and finally for a well advanced and successful trial operation. It is also a good opportunity for the authors to acknowledge Dr. S. Meigo and his group members of J-PARC MLF for always cooperating well during the RCS beam studies and measuring extracted beam profiles in the present experiment as well. Fruitful discussion and direct support from Dr. Y. Shobuda of J-PARC and Dr. J. A. Holmes of SNS concerning ORBIT code development and enhancement for beam simulations in the RCS are also highly appreciated.

[1] High-intensity Proton Accelerator Project Team, JAERI Report No. JAERI-Tech 2003-044 and KEK Report No. 2002-13.

[2] M. Kinsho, in Proceedings of the 2013 International Particle Accelerator Conference, IPAC'13, Shanghai, China, 2013, pp. 3848-3850.

[3] H. Hotchi, H. Harada, N. Hayashi, M. Kinsho, P. K. Saha, Y. Shobuda, F. Tamura, K. Yamamoto, M. Yamamoto, M. Yoshimoto, Y. Irie, and S. Kato, in Proceedings of the 2013 International Particle Accelerator Conference, IPAC'13, Shanghai, China, 2013 (Ref. [2]), pp. 3836-3838.

[4] P. K. Saha, Y. Shobuda, H. Hotchi, N. Hayashi, T. Takayanagi, H. Harada, and Y. Irie, Phys. Rev. ST Accel. Beams 12, 040403 (2009).

[5] F. Tamura, M. Yamamoto, M. Yoshii, C. Ohmori, M. Nomura, A. Schnase, M. Toda, H. Suzuki, T. Shimada, K. Hara, and K. Hasegawa, Phys. Rev. ST Accel. Beams 12, 041001 (2009).

[6] H. Hotchi, H. Harada, N. Hayashi, M. Kinsho, P. K. Saha, Y. Shobuda, F. Tamura, K. Yamamoto, M. Yamamoto, and M. Yoshimoto, Phys. Rev. ST Accel. Beams 15, 040402 (2012).
[7] P. K. Saha, S. Hatakeyama, K. Yamamoto, M. Yoshimoto, H. Harada, H. Hotchi, Y. Shobuda, M. Kinsho, K. Satou, and Y. Irie, Phys. Rev. ST Accel. Beams 14, 072801 (2011).

[8] Kazami Yamamoto and Shinichi Kato, Phys. Rev. ST Accel. Beams 15, 120401 (2012).

[9] Shinichi Kato, Kazami Yamamoto, Masahiro Yoshimoto, Hiroyuki Harada, and Michikazu Kinsho, Phys. Rev. ST Accel. Beams 16, 071003 (2013).

[10] N. Hayashi, H. Harada, K. Horino, H. Hotchi, J. Kamiya, M. Kinsho, P. K. Saha, Y. Shobuda, T. Takayanagi, N. Tani, T. Togashi, T. Ueno, M. Watanabe, Y. Watanabe, K. Yamamoto, M. Yamamoto, Y. Yamazaki, M. Yoshimoto, Y. Irie, and T. Toyama, in Proceedings of the 2013 International Particle Accelerator Conference, IPAC'13, Shanghai, China, 2013 (Ref. [2]), pp. 3833-3835.

[11] T. Takayanagi, N. Hayashi, T. Ueno, K. Horino, T. Togashi, K. Kinsho, Y. Watanabe, and Y. Irie, in Proceedings of the 2013 International Particle Accelerator Conference, IPAC'13, Shanghai, China, 2013 (Ref. [2]), pp. 681-683.

[12] N. Tani et al., in Proceedings of the 2013 International Conference on Magnet Technology, MT 23, Boston, MA, USA, 2013 [IEEE Trans. Appl. Supercond. (to be published)].

[13] Hideaki Hotchi et al., Prog. Theor. Exp. Phys. 2012, 02 B003 (2012).

[14] Strategic Accelerator Design, SAD, see http://acc-physics .kek.jp/SAD.

[15] H. Hotchi, H. Harada, N. Hayashi, M. Kinsho, P. K. Saha, Y. Shobuda, F. Tamura, K. Yamamoto, M. Yamamoto, M. Yoshimoto, and Y. Irie, in Proceedings of the 52nd ICFA Advanced Beam Dynamics Workshop, HB2012, Beijing, China, 2012, pp. 68-72.

[16] J. Kamiya, M. Kinsho, N. Hayashi, H. Hotchi, N. Ogiwara, N. Tani, and Y. Watanabe, in Proceedings of the 2013 International Particle Accelerator Conference, IPAC'13, Shanghai, China, 2013 (Ref. [2]), pp. 3327-3329.

[17] J.A. Holmes, in Proceedings of the 2010 International Particle Accelerator Conference, IPAC'10, Kyoto, Japan, 2010 (ICR, Kyoto, 2010), pp. 1901-1903.

[18] P. K. Saha, Y. Shobuda, H. Hotchi, H. Harada, M. Yamamoto, J. A. Holmes, and S. Kato, in Proceedings of the 2013 International Particle Accelerator Conference, IPAC'13, Shanghai, China, 2013 (Ref. [2]), pp. 521-523. 